\title{
Triangular Vertex Transform Based Watermarking on Contourlet Coefficients for Improved Robustness
}

\author{
Reena Thomas ${ }^{\mathrm{a}}$, Sucharitha $\mathbf{M}^{\mathrm{b}}$ \\ a Depatrment of Electronics and Communication Engineering, Noorul Islam Centre for Higher Education, Kumaracoil, India, \\ reenaresearch@gmail.com \\ ${ }^{\mathrm{b}}$ Department of Electronics and Communication Engineering, Malla Reddy College of Engineering and Technology, \\ Hyderabad, India
}

Article History: Received: 10 November 2020; Revised 12 January 2021 Accepted: 27 January 2021; Published online: 5 April 2021

\begin{abstract}
A hybrid watermarking scheme based on Triangular Vertex Transform (TVT) and Contourlet coefficients for high robustness is implemented. During watermark embedding, the cover image is first decomposed using Contourlet Transform to obtain high frequency and low frequency coefficients. The lower frequency coefficients are applied with TVT. Then, the W coefficients obtained from TVT are again subdivided. The watermark bit is then embedded on the subdivided coefficients to obtain the watermarked image. Reverse operation is followed in the extraction phase. The performance of this algorithm is evaluated using embedding capacity, Normalized cross correlation ( $\mathrm{Ncc}$ ) and Peak Signal to Noise Ratio (PSNR) using standard test images. These evaluation results disclose the domination of proposed scheme over traditional schemes.
\end{abstract}

Keywords: Contourlet transform, Triangular vertex transform, Watermarking, Robustness, Coefficients, Hybrid watermarking

\section{Introduction}

Due to the development of high speed internet, huge number of media like images are created, manipulated, and shared through it especially in social media. Therefore it is very necessary to preserve the ownership of media like images. Digital image watermarking [1] provides a way to preserve the ownership of media, which also have other applications in medical field and military. A good watermarking algorithm must have the properties like high embedding capacity, high visual quality and robustness against attacks. Several researchers are working in developing high capacity, high visual quality and highly robust algorithms. Spatial domain schemes [2] directly embed the data on the pixel intensities. Schemes on transform domain convert the pixels intensities into coefficients for embedding the data.

Spatial domain methods provide high quality images and the robustness against attacks is less. Transform domain schemes are introduced to accomplish elevated robustness. These schemes mainly use Fourier Transform, Discrete Wavelet Transform (DWT) and Discrete Cosine Transform (DCT). These transforms usually generate a collection of coefficients having different frequency components. Apart from transform domain methods, decomposition methods like Singular Value Decomposition (SVD), QR decomposition and LU decomposition are used. Hybrid schemes [3] were also introduced to improve the robustness of embedded image. Hybrid schemes uses two or more transforms with decomposition algorithms. Kalra et al. [4] used DCT coefficients on each blocks of the image and the blocks were selected based on the variance. Jane et al. [5] used DWT with LU and SVD. Su et al. [6] used Schur decomposition for embedding the data on first column elements on second and third row. This shows that the robustness and PSNR also depends on the position of coefficients in which data is embedded. Rai et al. [7] used the watermarking scheme for medical applications, where a key is used to scramble the data. This ensures the security in image watermarking. Su et al. [8] used LU decomposition in which lower and upper triangular matrices were estimated after sub-dividing the image or coefficients to $4 \times 4$ blocks. The lower triangular matrix was used in embedding the data.

This work focus on improving the robustness and PSNR of color image watermarking. The paper formulates a hybrid algorithm that use Contourlet Transform (CT) [9] and Triangular Vertex Transform (TVT) [10] which is robust against attacks. The forthcoming sections of the paper are arranged in the following manner. Section 2 shows the proposed watermarking algorithm. Section 3 explains the experimental results and validation of the algorithm and finally conclusion is provided in section 4.2 . 


\section{Methodology}

Figure 1 depicts the functional block diagram of watermark embedding process. Initially, the cover image $I$ is separated into red $\left(I_{R}\right)$, green $\left(I_{G}\right)$ and blue $\left(I_{B}\right)$ components. CT is applied on these components to obtain Contourlet coefficients. $C_{R}, C_{G}$ and $C_{B}$ are the Contourlet coefficients that are applied to the TVT. Then TVT generate $U, V$ and $W$ coefficients. Among these coefficients, $W$ is used for embedding the watermark. For embedding the watermark, $W$ is partitioned into $4 \times 4$ sub-blocks. $F$ represents the sub-block where the binary data $b$ is embedded to obtain the embedded block $F^{\prime}$. After embedding the data into each sub-block, they are merged together to obtain $W^{\prime}$. Then Inverse Triangular Vertex Transform (ITVT) is applied using the $U, V$ and $W^{\prime}$ coefficients to obtain $C_{R}^{\prime}, C_{G}^{\prime}$ and $C_{B}^{\prime}$. Using the coefficients $C_{R}^{\prime}$ and $D_{R}$, it is possible to estimate the red channel of the watermarked image $I_{R}^{\prime}$. Similarly, it is possible to estimate $I_{G}$ and $I_{B}, R, G$ and $B$ planes applied with watermark are merged together to generate the final watermarked image $I^{\prime}$.

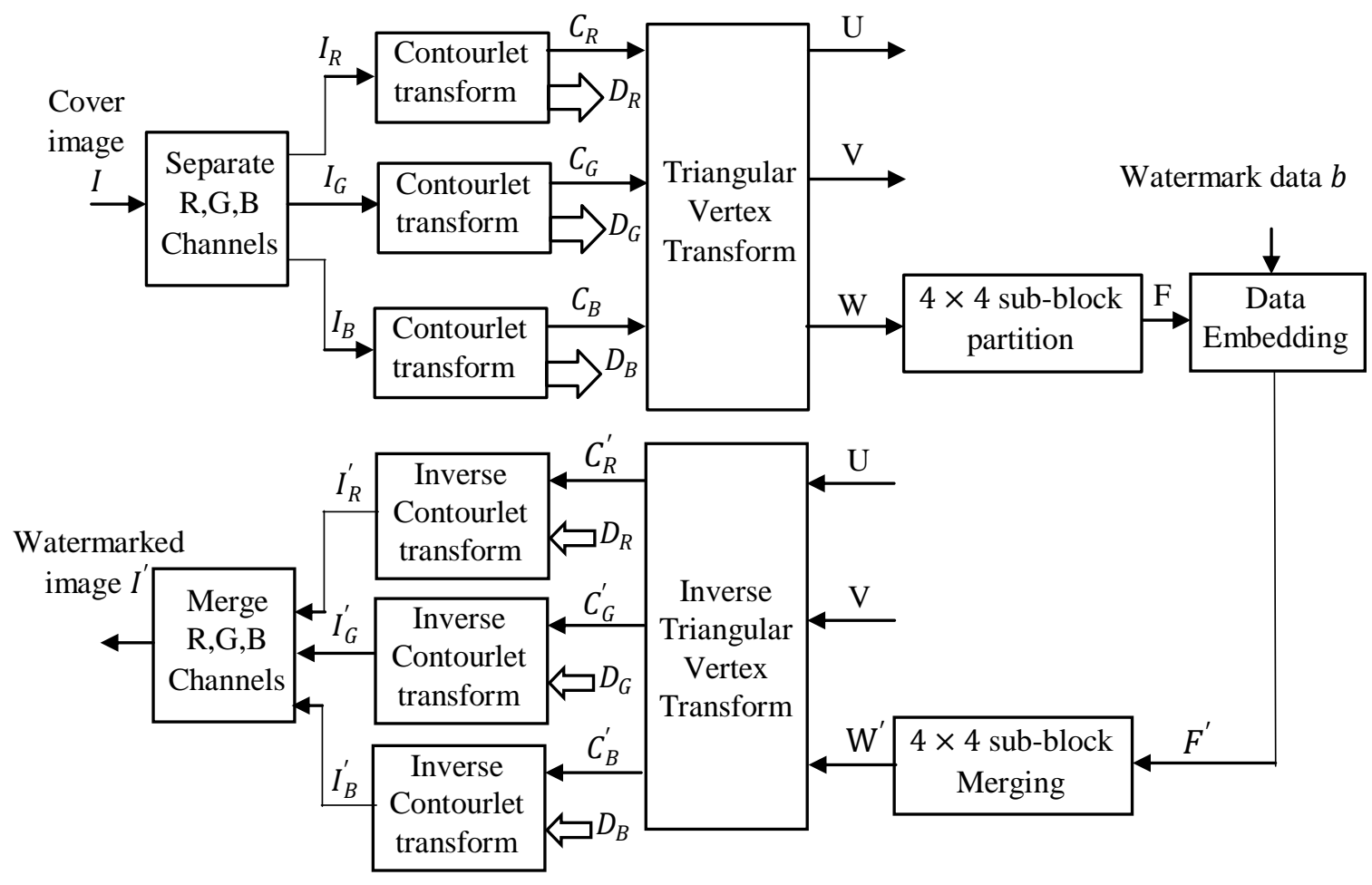

Figure 1: Watermark embedding process

The main advantage of Contourlet Transform [9] is that, it can preserve the geometry of edges present in the image. In this transform, discrete multi-resolution coefficients that represent the diagonal coefficients are generated using non-separable filter banks. This transform has properties such as ability to reconstruct and orthogonality. The $l$ directional filter bank can be represented as $d_{k}(l), 0 \leq k \leq 2^{l}-1$. The value of $l$ decides the number of sub-bands. A channel $g^{(n)}$ is applied to the input of Laplacian pyramid which provides $m$ number of band pass images represented by $z_{m}^{(n)}$. The coarser image of $z_{m-1}^{(n)}$ be $z_{m}^{(n)}$ and the image $g^{(n)}$ can be therefore decomposed using directional filter bank with $l_{m}$ levels. Here we use the number of levels as 2 .

The coefficients $U, V$ and $W$ are estimated by assigning the Contourlet coefficients $C_{R}, C_{G}$ and $C_{B}$ as the edges of a triangle. Using these edges, the vertices are estimated as $\mathrm{U}, \mathrm{V}$ and $\mathrm{W}$ coefficients using the following equations,

$$
\begin{aligned}
& U=\frac{C_{G}^{2}-C_{B}^{2}+C_{R}^{2}}{2 C_{R}} \\
& V=\sqrt{C_{G}^{2}-\frac{C_{G}^{2}-C_{B}^{2}+C_{R}^{2}}{2 C_{R}}}
\end{aligned}
$$




$$
W=\frac{C_{R}^{2}-C_{G}^{2}+C_{B}^{2}}{2 C_{R}}
$$

The inverse TVT transform will converts the $\mathrm{U}, \mathrm{V}$, and W coefficients back to the contourlet coefficients $C_{R}$, $C_{G}$ and $C_{B}$ represented by the following equations,

$$
\begin{aligned}
& C_{R}=U+W \\
& C_{G}=\sqrt{V^{2}+U^{2}} \\
& C_{B}=\sqrt{V^{2}+W^{2}}
\end{aligned}
$$

If the coefficients $C_{R}, C_{G}$ and $C_{B}$ are same $\left(C_{R}=C_{G}=C_{B}\right)$, then the coefficients are modified as,

$$
C_{R} \leftarrow C_{R}-\beta / 2 ; C_{G} \leftarrow C_{G} ; C_{B} \leftarrow C_{B}+\beta / 2
$$

Let the $4 \times 4 \mathrm{~W}$-coefficients be represented as,

$$
F=\left[\begin{array}{cccc}
f_{11} & f_{12} & f_{13} & f_{14} \\
f_{21} & f_{22} & f_{23} & f_{24} \\
f_{31} & f_{32} & f_{33} & f_{34} \\
f_{41} & f_{42} & f_{43} & f_{44}
\end{array}\right]
$$

The coefficients after data embedding is represented as,

$$
\begin{aligned}
& F^{\prime}=\left[\begin{array}{cccc}
f_{11}^{\prime} & f_{12}^{\prime} & f_{12}^{\prime} & f_{14} \\
f_{21} & f_{22} & f_{23} & f_{24} \\
f_{31} & f_{32} & f_{33} & f_{34} \\
f_{41} & f_{42} & f_{43} & f_{44}
\end{array}\right] \\
& f_{11}^{\prime}=\operatorname{sign}\left(f_{11}\right) \times\left(\left|f_{11}\right|-0.25 \alpha \Delta\left(\left|f_{11}\right|+\left|f_{13}\right|\right)\right) \\
& f_{12}^{\prime}=0.5 \times \operatorname{sign}\left(f_{12}\right)\left(\left|f_{11}\right|+\left|f_{13}\right|\right)(1+\alpha \Delta) \\
& f_{13}^{\prime}=\operatorname{sign}\left(f_{13}\right) \times\left(\left|f_{13}\right|-0.25 \alpha \Delta\left(\left|f_{11}\right|+\left|f_{13}\right|\right)\right)
\end{aligned}
$$

where, $\operatorname{sign}(x)=\left\{\begin{array}{c}0 x=0 \\ -1 x<0 \\ 1 x>0\end{array}\right.$

The value of $\Delta$ can be estimated as, 


$$
\Delta=\left\{\begin{array}{lll}
-1 & \text { if } b=0 \\
1 & \text { if } b=1
\end{array}\right.
$$

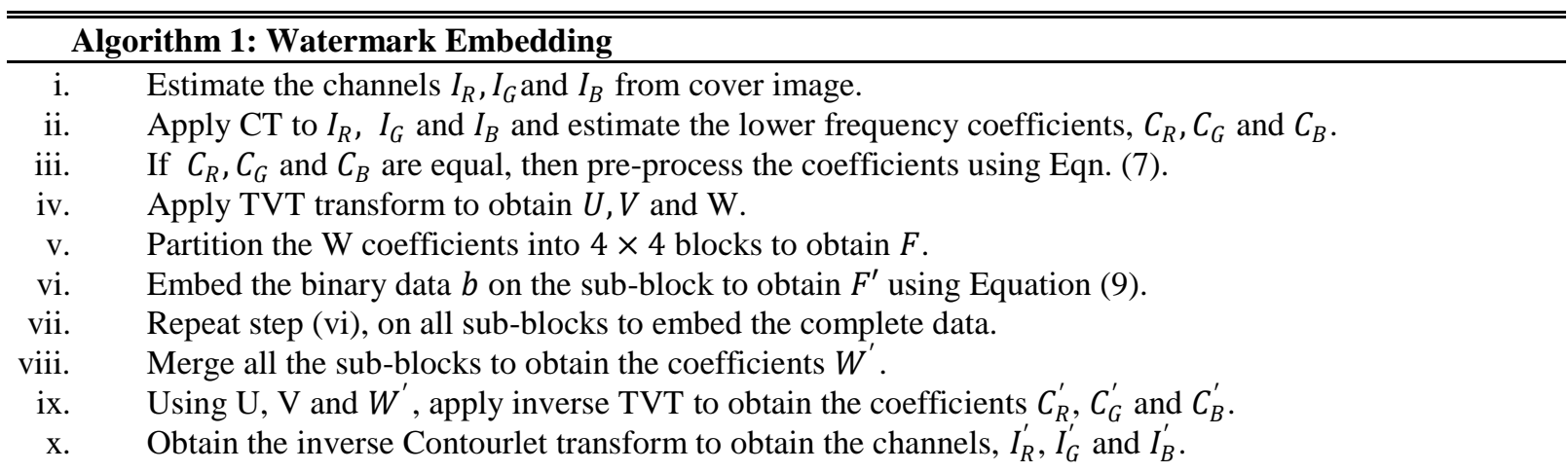

During the extraction process, the watermarked image is initially separated into $\hat{I}_{R}, \hat{I}_{G}$ and $\hat{I}_{B}$ as depicted in Figure 2. From each channels, the low frequency Contourlet coefficients are estimated as $\hat{C}_{R}, \hat{C}_{G}$ and $\hat{C}_{B}$.

Using TVT, the coefficients $\widehat{U}, \widehat{V}$ and $\widehat{W}$ are estimated and $\widehat{W}$ is sub-divided into $4 \times 4$ blocks represented as $\widehat{F}$,

$$
\hat{F}=\left[\begin{array}{llll}
\hat{f}_{11} & \hat{f}_{12} & \hat{f}_{13} & \hat{f}_{14} \\
\hat{f}_{21} & \hat{f}_{22} & \hat{f}_{23} & \hat{f}_{24} \\
\hat{f}_{31} & \hat{f}_{32} & \hat{f}_{33} & \hat{f}_{34} \\
\hat{f}_{41} & \hat{f}_{42} & \hat{f}_{43} & \hat{f}_{44}
\end{array}\right]
$$

The data $\hat{b}$ present in the sub-block $\hat{F}$ is extracted using the following relation.

$$
\hat{b}=\left\{\begin{array}{rr}
1 & \left|\hat{f}_{12}\right|>0.5\left(\left|\hat{f}_{11}\right|+\left|\hat{f}_{13}\right|\right) \\
0 & \text { elsewhere }
\end{array}\right.
$$

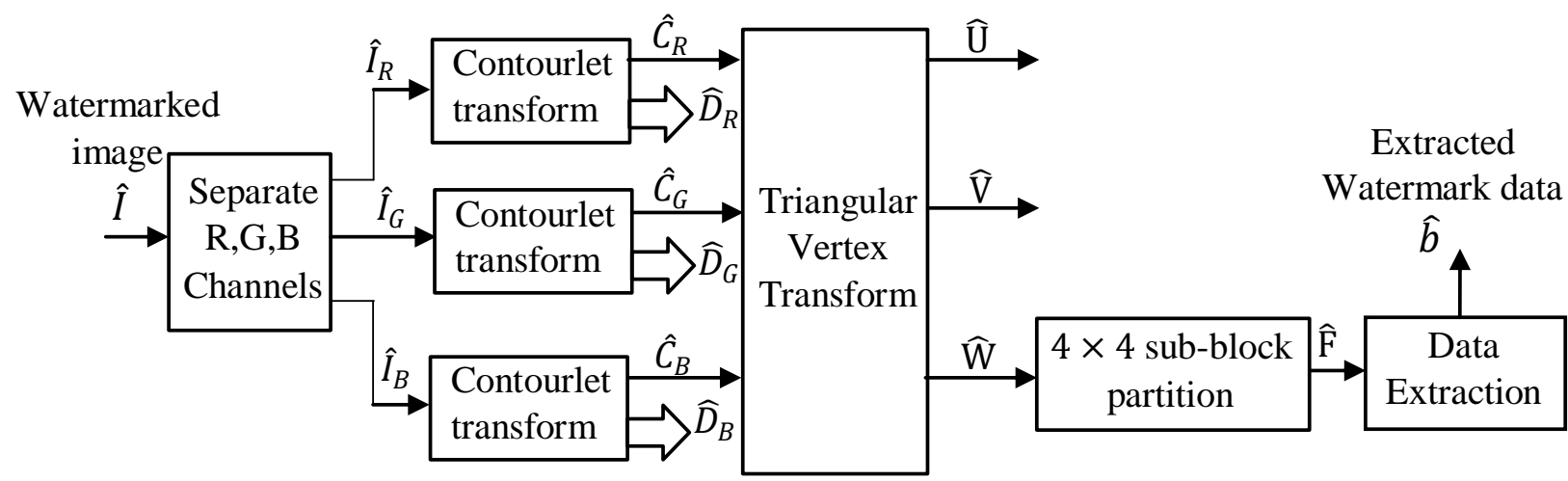

Figure 2: Watermark extraction process

The procedure for watermark extraction is briefed in algorithm 2 . 


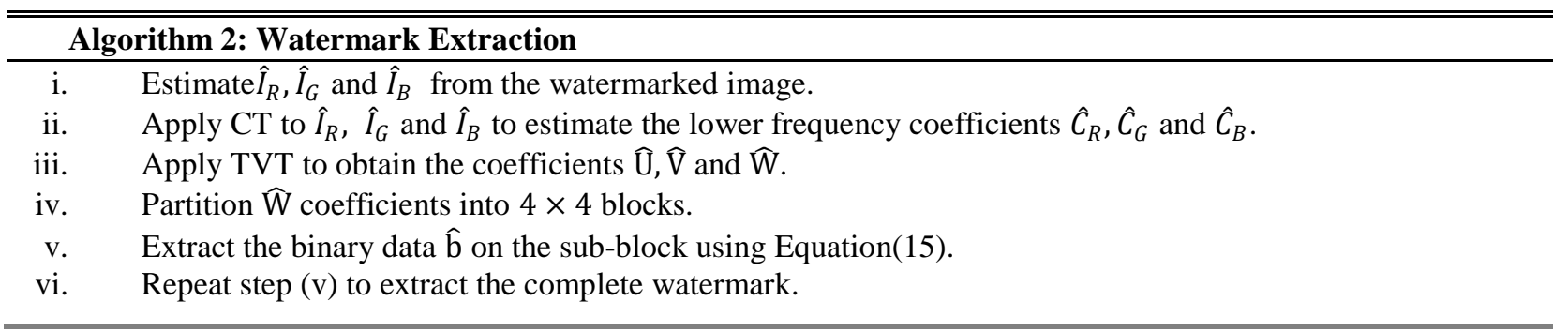

\section{Experimental Results}

Performance of the proposed algorithm is evaluated using standard test images such Airplane, Baboon, Barbara, Fruits, Lena, Pepper and Tifffany. These standard color (RGB) images have dimension $512 \times 512$ as depicted in Figure 3. The watermark images are binary images having a size of $64 \times 64$ as shown in Figure 4 . The performance is evaluated using Embedding Capacity (EC), PSNR, Structural Similarity Index Measurement (SSIM) and Ncc. Table 1 displays the comparison of performance for different test images. The proposed scheme provides a maximum EC of 4096 bits. The value of SSIM and PSNR are highest for the test image Tiffany.

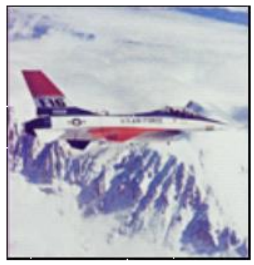

(a)

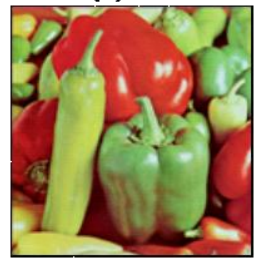

(d)

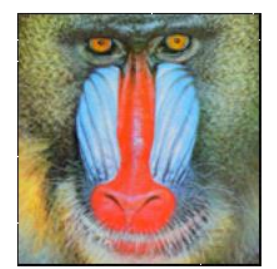

(b)

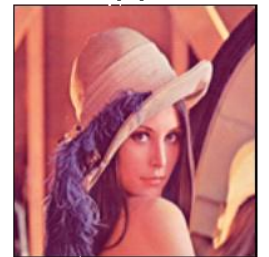

(e)

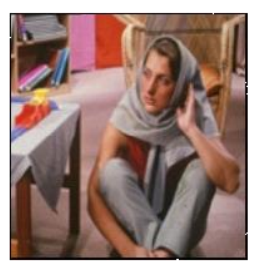

(c)

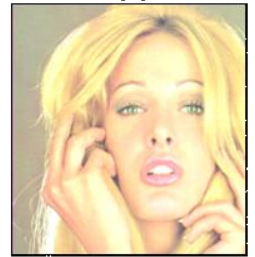

(f)

Figure 3: Test images (a) Airplane (b) Baboon

(c) Barbara (d) Pepper (e) Lena (f) Tiffany

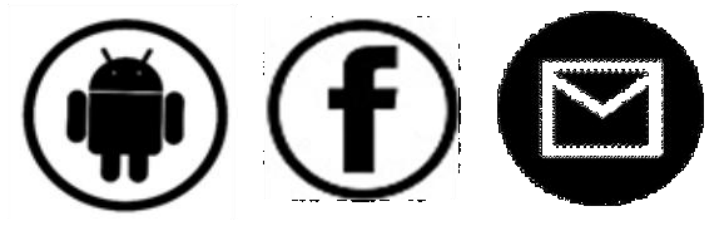

Figure 4: Test Secret images (a) Android (b) Facebook (c) Gmail 
Table 1: Performance of the proposed method for different test images.

\begin{tabular}{rlrrrrrrr}
\hline \multirow{2}{*}{ Watermark } & \multirow{2}{*}{ Metric } & \multicolumn{9}{c}{ RGB Host image } \\
& & Airplane & Baboon & Barbara & \multicolumn{1}{c}{ Fruit } & \multicolumn{1}{c}{ Lena } & Pepper & Tiffany \\
\hline \multirow{3}{*}{ Android } & PSNR(dB) & 49.4091 & 42.6593 & 46.4111 & 45.0576 & 48.6623 & 41.9953 & 50.7294 \\
& SSIM & 0.9991 & 0.9986 & 0.9987 & 0.9981 & 0.9991 & 0.9959 & 0.9989 \\
& Ncc & 0.9994 & 0.9940 & 1 & 0.988 & 1 & 0.9448 & 0.9974 \\
Facebook & SSNR(dB) & 49.0441 & 42.8751 & 46.5749 & 45.2468 & 48.4034 & 42.0465 & 50.8291 \\
& Ncc & 0.9991 & 0.9987 & 0.9988 & 0.9981 & 0.9990 & 0.9959 & 0.9989 \\
& PSNR(dB) & 0.9993 & 0.9938 & 0.9998 & 0.9886 & 1.000 & 0.9555 & 0.9983 \\
Gmail & SSIM & 0.5420 & 43.4300 & 47.5914 & 45.6544 & 48.7772 & 42.4312 & 50.8964 \\
& Ncc & 0.9991 & 0.9987 & 0.9990 & 0.9982 & 0.9991 & 0.9962 & 0.9989 \\
& & & 0.9934 & 1.0000 & 0.9816 & 1.0000 & 0.9535 & 0.9880 \\
\hline
\end{tabular}
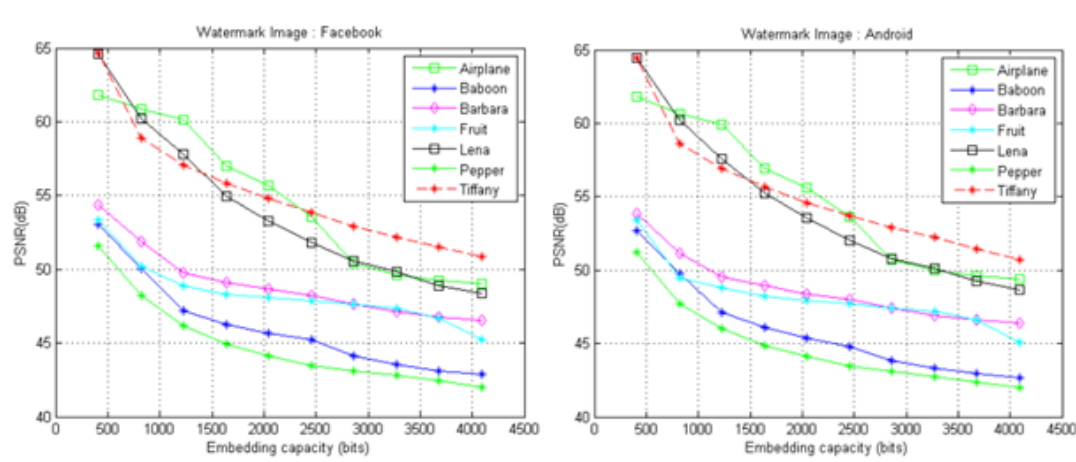

(b)

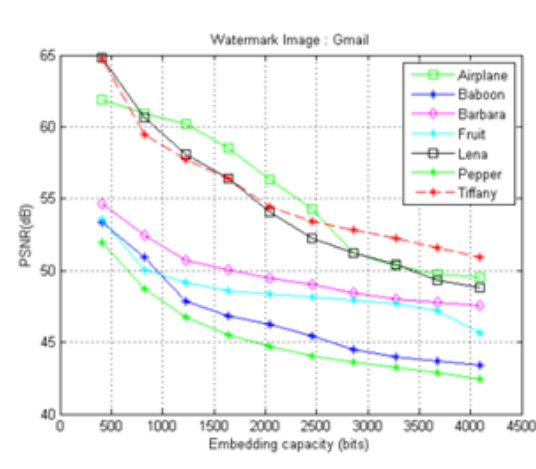

(c)

Figure 5: PSNR Vs embedding capacity (a) Facebook (b) Android (c) Gmail

Figure 5 shows the graphical comparison of PSNR for different embedding capacity. As the value of EC increases, PSNR decreases. PSNR is high for Tiffany image and it is less for the Pepper image almost for different embedding capacity. PSNR is almost independent on the data which is embedded. Fig 6 shows the PSNR and Ncc comparison for different values of $\alpha$. As the value of $\alpha$ is less, PSNR is almost same for all values of $\beta$. For higher values of $\alpha$, PSNR is more dependent of the value of $\beta$. Similarly, the Ncc is independent of $\beta$ for $\alpha=0.5$. As the value of $\propto$ increases, Ncc is more dependent of the value of $\beta$. Fig 7 shows the extracted watermark for various attacks.
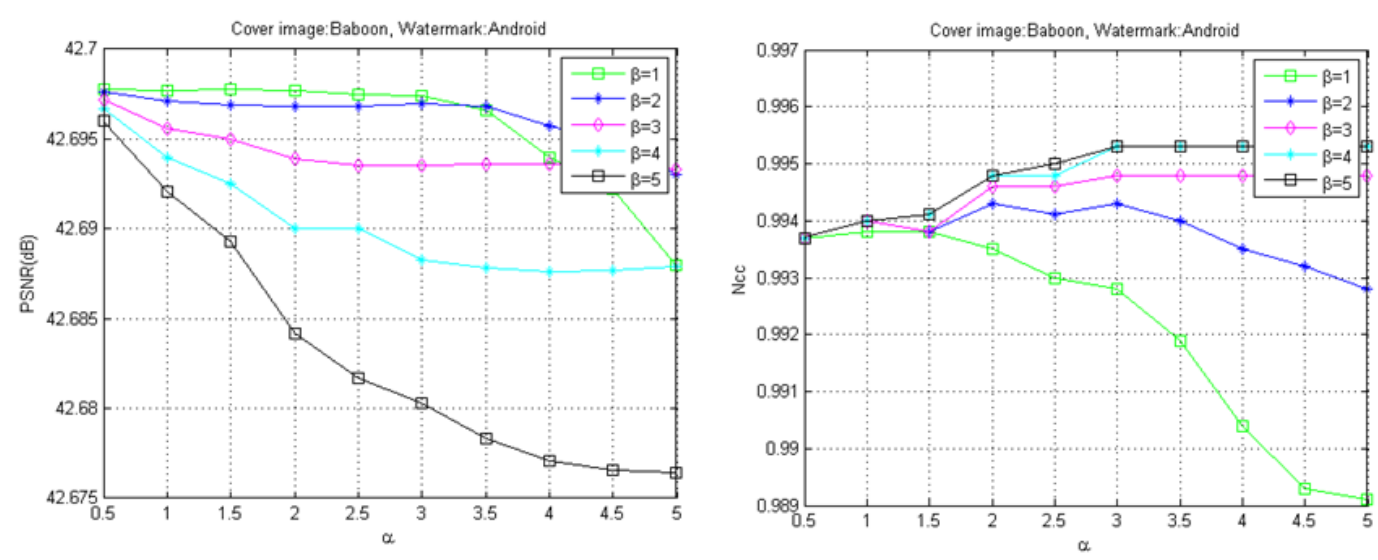
Figure 6: PSNR and Ncc comparison for different values of $\propto$ (a) PSNR (b) Ncc

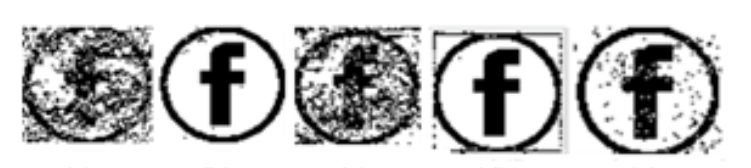

(a)

(b)

(c)

(d)

(e)

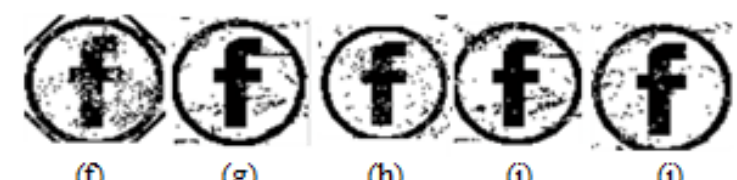

(f)

(g)

(h)

(i)

(j)

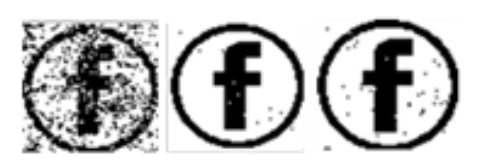

(k)

(1)

(m)

Figure 7: Extracted watermark after attacks (a) Histogram Equalization (b) Brightening (c) Sharpening (d) Salt \& pepper noise (e) Cropping (f) Rotation (g) Darkening (h) JPEG 2000 (i) Contrast adjustment (j) Gamma correction (k) Median filtering (l) Scaling (m) Gaussian LPF.

Table 2: PSNR and SSIM comparison

\begin{tabular}{lll}
\multicolumn{2}{l}{ Method PSNR(dB) } & SSIM \\
Scheme [11] & 42.31 & 0.9801 \\
Scheme [12] & 43.44 & 0.9806 \\
Scheme [13] & 44.63 & 0.9831 \\
Scheme [10] & 45.23 & 0.9852 \\
Proposed & 46.58 & 0.9991
\end{tabular}

Table 2 provides a comparison of SSIM and PSNR with tradional methods. The average PSNR of proposed scheme is $46.58 \mathrm{~dB}$, which is higher compared to tradional methods [13], [12], [11] and [10]. Also, the proposed schemes provides a SSIM of 0.9991 which is also higher than the traditional schemes. Figure 8 depicts the PSNR comparison for different values of $\beta$. Figure 9 shows the Ncc comparison for various $\beta$ values.

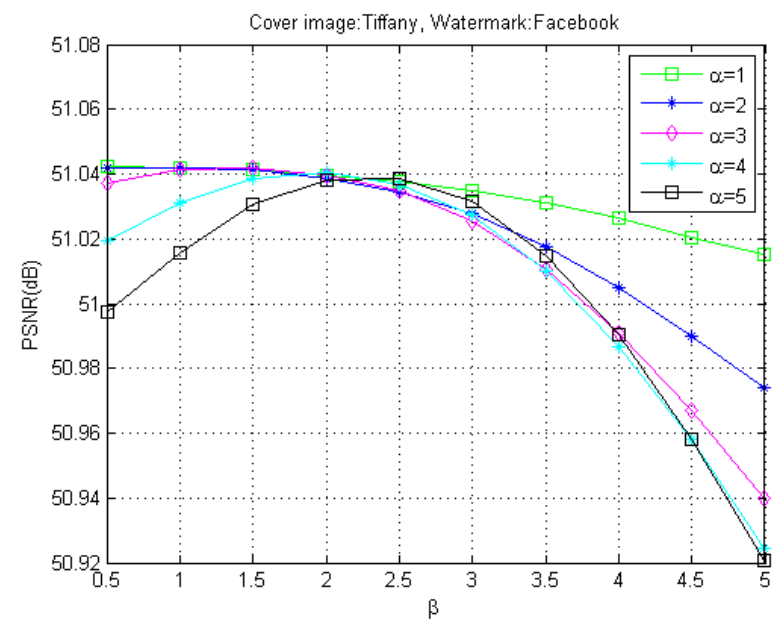


Figure 8: PSNR for different values of $\beta$

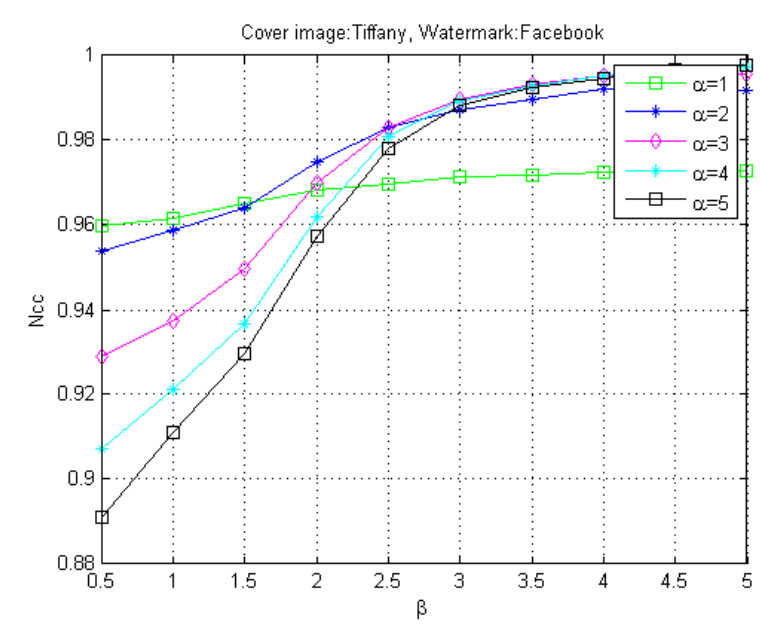

Figure 9: Ncc for different values of $\beta$

Table 3: Ncc for different test images under attack

\begin{tabular}{lccc}
\hline \multicolumn{1}{c}{ Attack } & $\begin{array}{c}\text { Barbara \& } \\
\text { Facebook }\end{array}$ & $\begin{array}{c}\text { Airplane } \\
\text { \& Android }\end{array}$ & $\begin{array}{c}\text { Fruit } \\
\& \text { Gmail }\end{array}$ \\
\hline Histogram Equalization & 0.7622 & 0.7519 & 0.5186 \\
Brightening & 0.9968 & 0.9932 & 0.8403 \\
Sharpening & 0.7862 & 0.9123 & 0.7374 \\
Salt \& Pepper noise & 0.8008 & 0.7840 & 0.6717 \\
Cropping & 0.9636 & 0.9620 & 0.8968 \\
Rotation & 0.9012 & 0.9038 & 0.8363 \\
Darkening & 0.9620 & 0.9651 & 0.9159 \\
JPEG 2000 & 0.9655 & 0.9192 & 0.9065 \\
Contrast Adjustment & 0.9517 & 0.8319 & 0.8386 \\
Gamma Correction & 0.9677 & 0.9359 & 0.7709 \\
Median Filtering & 0.8586 & 0.7803 & 0.6368 \\
Scaling & 0.9977 & 0.9840 & 0.9612 \\
Gaussian LPF & 0.9930 & 0.9856 & 0.9511 \\
\hline
\end{tabular}

For $\beta=2$, PSNR is independent on the value of $\alpha$. Ncc will be high for higher values of $\beta$. Therefore, for achieving high PSNR, $\alpha$ can be chosen as minimum and $\beta$ as 2. For high robustness moderate value of $\alpha$ and higher values of $\beta$ need to be chosen. Table 3 displays the Ncc comparison for various types of attacks. Table 4 displays the Ncc comparison with the traditional schemes. For most of the attacks, Ncc of the proposed method is higher than that of existing schemes. This is the indication of the robustness against various attacks.

Table 4: Ncc Comparison of proposed method with traditional schemes

\begin{tabular}{lccccc}
\hline \multicolumn{1}{c}{ Attack } & $\begin{array}{c}\text { Scheme } \\
{[11]}\end{array}$ & $\begin{array}{c}\text { Scheme } \\
{[12]}\end{array}$ & $\begin{array}{c}\text { Scheme } \\
{[13]}\end{array}$ & $\begin{array}{c}\text { Scheme } \\
{[10]}\end{array}$ & Proposed \\
\hline Histogram & 0.8654 & 0.9122 & 0.9017 & 0.9186 & \\
Equalization & & & & & 0.6776 \\
Brightening & 0.7822 & 0.9276 & 0.9253 & 0.9327 & 0.9434 \\
Sharpening & 0.8542 & 0.9342 & 0.9412 & 0.9596 & 0.8120 \\
Salt \& Pepper noise & 0.7432 & 0.8634 & 0.9321 & 0.9431 & 0.7522
\end{tabular}




\begin{tabular}{llllll} 
Cropping & 0.8532 & 0.9024 & 0.9125 & 0.9238 & 0.9408 \\
Rotation & 0.6285 & 0.6645 & 0.8678 & 0.8745 & 0.8804 \\
Darkening & 0.7822 & 0.9176 & 0.9253 & 0.9399 & 0.9477 \\
JPEG 2000 & 0.9132 & 0.9222 & 0.9184 & 0.9054 & 0.9304 \\
Contrast Adjustment & 0.7072 & 0.8563 & 0.8522 & 0.8699 & 0.8741 \\
Gamma Correction & 0.8835 & 0.8432 & 0.8821 & 0.8732 & 0.8915 \\
Median Filtering & 0.8602 & 0.8751 & 0.9264 & 0.9143 & 0.7586 \\
Scaling & 0.8758 & 0.8543 & 0.8902 & 0.9784 & 0.9810 \\
Gaussian LPF & 0.9214 & 0.9564 & 0.9372 & 0.9532 & 0.9766 \\
\hline
\end{tabular}

\section{Conclusion}

This work introduced a watermarking scheme that utilizes transforms such as CT and TVT. The cover image is applied with CT to obtain the lower frequency coefficients which is again transformed using TVT. The W coefficient of TVT is used for embedding watermark. The experimental evaluation is performed on standard test images using metrics such as EC, SSIM, Ncc and PSNR. Average PSNR obtained is $46.58 \mathrm{~dB}$ at the embedding capacity is 4096 bits. The proposed scheme is found to be robust against various attacks. This will improve the security of watermark while transmitting though a communication channel.

\section{References}

J. Li, C. Yu, B. Gupta, X. Ren, "Color Image Watermarking Scheme Based on Quaternion Hadamard Transform and Schur Decomposition". Multimedia Tools and Applications, Vol.77, pp.4545-4561, 2018.

P. Parashar P, R.K. Singh, "A Survey: Digital Image Watermarking Techniques". Int J Signal Process Image Process and Pattern Recognit, Vol.7, pp.111-124, 2014.

A.K. Singh, "Improved hybrid algorithm for robust and imperceptible multiple watermarking using digital images". Multimedia Tools and Applications, Vol.76, pp.8881-8900, 2017.

G.S. Kalra, R. Talwar, H. Sadawarti "Adaptive digital image watermarking for color images in frequency domain". Multimedia Tools and Applications, Vol.74, pp. 6849-6869, 2015.

O. Jane, E. Elbasi, "A new approach of nonblind watermarking methods based on DWT and SVD via LU decomposition". Turkish Journal of Electronics Engineering and Computer Science, Vol.22, No.1, pp. 13541366, 2014.

Q. Su, Y. Niu, X. Liu, Y. Zhu, "Embedding color watermarks in color images based on Schur decomposition". Optical Communication, Vol. 285, No.1, pp. 1792-1802, 2012.

A. Rai, H.V. Singh, "SVM based robust watermarking for enhanced medical image security". Multimedia Tools and Applications, Vol.76, pp. 18605-18618, 2017.

Q. Su, G. Wang, X. Zhang, G. Lv, B. Chen, "A new algorithm of blind color image watermarking based on LU decomposition", Multidimensional Systems and Signal Processing, Vol.29, No.3, pp. 1055-1074, 2018.

A. Akhaee, S.M. Sahraeian, F. Marvasti, "Contourlet-based image watermarking using optimum detector in noisy environment". IEEE Transaction on Image Processing, Vol.9, No.4, pp. 700-715, 2010.

K. Prabha, I. Shatheesh Sam. "A novel blind color image watermarking based on Walsh Hadamard Transform". Multimedia Tools and Applications Vol.79, pp. 6845-6869, 2020.

Q. Su, Y. Niu Y, Q. Wang, G. Sheng, "A blind color image watermarking based on DC component in the spatial domain”. Optik, Vol.124, pp. 6255-6260, 2013.

N. Liu, H. Li, H. Dai, "Robust blind image watermarking based on chaotic mixtures". Nonlinear Dynamics Vol.80, No.3, pp. 1329-1355, 2015.

H. Cheng HS, Y. Feng, R. Wang, J. Min, "A robust color image watermarking algorithm against rotation attacks". Optoelectronics Letters, Vol.14, pp. 61-66, 2018. 